\title{
Resultados de lesión del nervio laríngeo recurrente en cirugía de tiroides con el uso del neuroestimulador
}

\author{
Results of injurious laryngeal recurrent in thyroid surgery with the use of the \\ neuroestimulator \\ Marina Jiménez-Segovia*, Cristina Álvarez-Segurado, Jaime Bonnín-Pascual, \\ Alessandro Bianchi y Xavier González-Argente \\ Departamento de Cirugía General y del Aparato Digestivo, Hospital Son Espases, Palma de Mallorca, España
}

\begin{abstract}
Resumen
Antecedentes: La neuromonitorización intraoperatoria intermitente del nervio laríngeo recurrente es el complemento ideal en las cirugías tiroideas, ya que disminuye las lesiones. Objetivo: Analizar la prevalencia de lesiones del nervio laríngeo recurrente con y sin el uso de neuromonitorización en cirugía de tiroides. Método: Estudio observacional, descriptivo y retrospectivo, en el que se incluyeron 571 pacientes entre los años 2012 y 2018. De ellos, en 180 se utilizó neuromonitorización y en 391 no. Resultados: De los 180 pacientes que se sometieron a tiroidectomía total con neuromonitorización hubo 8 (4.4\%) parálisis transitorias y 2 (1.1\%) parálisis definitivas. Sin el uso de neuromonitorización hubo 12 (3\%) parálisis transitorias y 7 (1.85\%) definitivas. Conclusiones: Creemos que la neuromonitorización debe usarse sistemáticamente con la técnica habitual. Obtenemos resultados significativos con respecto a la reducción de las lesiones del nervio laríngeo recurrente con el uso de neuromonitorización intraoperatoria.
\end{abstract}

Palabras clave: Complicaciones postiroidectomía. Lesión nervio laríngeo recurrente. Neuroestimulador.

\begin{abstract}
Background: Intermittent intraoperative neuromonitoring of the recurrent laryngeal nerve is the ideal complement in thyroid surgeries, decreasing thyroid injuries. Objective: To analyze the prevalence of recurrent laryngeal nerve injuries with the use and without the use of neuromonitoring in thyroid surgery. Method: Observational, descriptive and retrospective study, in which a total of 571 patients were included between the years 2012-2018. Of which 180 neuromonitoring was used and 391 were not used. Results: Of the 180 patients who underwent total thyroidectomy with the use of neuromonitoring, we had a total of $8(4.4 \%)$ transient paralysis and $2(1.1 \%)$ definitive. Without the use of neuromonitoring we obtain 12 (3\%) transient paralysis and $7(1.85 \%)$ definitive. Conclusions: We believe that the use of neuromonitoring complementary to surgery should be used routinely to the usual technique. And we also obtain significant results regarding the reduction of recurrent laryngeal nerve injuries with the use of intraoperative neuromonitoring.
\end{abstract}

Key words: Postiroidectomy complications. Injury recurrent laryngeal nerve. Neurostimulator.

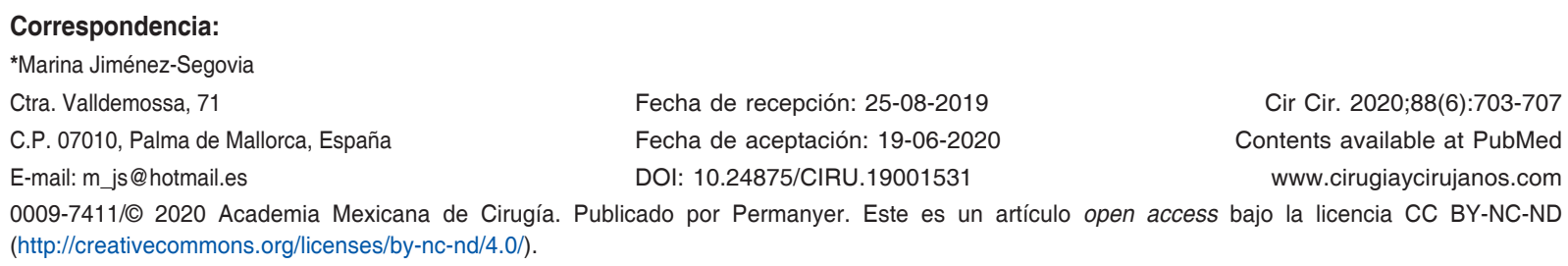

Cir Cir. 2020;88(6):703-707 Contents available at PubMed www.cirugiaycirujanos.com 0009-7411/@ 2020 Academia Mexicana de Cirugía. Publicado por Permanyer. Este es un artículo open access bajo la licencia CC BY-NC-ND (http://creativecommons.org/licenses/by-nc-nd/4.0/). 


\section{Introducción}

La lesión del nervio laríngeo recurrente (NLR) y el hipoparatiroidismo secundario son las complicaciones más significativas de la cirugía de tiroides, con un gran impacto en la calidad de vida del paciente e implicaciones de tipo médico-legal.

El NLR, debido a su vulnerabilidad, puede estar sujeto a lesiones de gran consecuencia funcional asociada, como alteraciones en la voz e incluso síntomas de disfagia con aspiración, y en caso de lesiones bilaterales puede llegar a insuficiencia respiratoria con necesidad de traqueostomía1. También se interpreta como un indicador de calidad que depende tanto de la enfermedad de la tiroides y del tipo de resección como del entrenamiento y la experiencia del cirujano ${ }^{2}$.

En otro orden de cosas, hay complicaciones técnicas durante la intervención que se derivan de la patología misma o de anomalías que pueden afectar la integridad del nervio: maniobras traumáticas que se realizan prematuramente por tracción, principalmente en la movilización de la glándula; isquemia por estiramiento o incluso por esqueletización del nervio; ligaduras hechas muy cerca de ella; sección nerviosa en situaciones extremas en las que el nervio no se ha identificado correctamente o se ha confundido con otra estructura, generalmente vascular, y en especialmente en circunstancias peligrosas debido al volumen de la glándula tiroides y su posición con la arteria tiroidea inferior; o cánceres infiltrativos con adherencias cercanas al ligamento de Berry ${ }^{3}$.

Desde Kocher hasta la descripción de la tiroidectomía moderna, los cirujanos intentan reducir las complicaciones asociadas con la lesión de las estructuras anatómicas involucradas en esta cirugía, con contribuciones fundamentales en la mejora de la técnica, en particular con la recomendación de identificar siempre el NLR ${ }^{4,5}$.

En las últimas dos décadas se han creado dispositivos para un mejor control intraoperatorio de la hemostasia, que ofrecen la posibilidad de mantener un campo operativo más exangüe y de esta manera facilitan la identificación de las estructuras anatómicas cercanas a la tiroides, y por lo tanto de las estructuras nerviosas.

Hay que tener en cuenta que existe otro riesgo de complicaciones dependiendo del tipo de sellador hemostático, y son las lesiones producidas por difusión electrotérmica de calor o contacto, así como el calentamiento por vibración de la película activa que emana de los dispositivos hemostáticos.

La neuromonitorización, en la práctica clínica, se ha introducido en las últimas dos décadas, aunque algunos dispositivos se crearon en los años 1970. Actualmente, después de años de controversia, pero destacando las ventajas de la identificación visual del NLR durante la tiroidectomía, se considera que la neuromonitorización constituye el complemento ideal para el método de referencia en las tiroidectomías y está siendo utilizado en los Estados Unidos por el $53 \%$ de los cirujanos generales y el $65 \%$ de los otorrinolaringólogos; en Europa, el $92 \%$ de los cirujanos alemanes lo usan de forma sistemática, tal como recomiendan las guías en su país ${ }^{6}$. La American Academy of Otolaryngology-Head and Neck Surgery considera que es especialmente útil en cirugías tiroideas bilaterales, revisiones de cirugía tiroidea y cirugía para parálisis previa del NLR?.

Los principales factores de riesgo para la lesión del NLR son antecedentes de disfonía, reoperaciones cervicales, enfermedad de Graves-Basedow, cánceres, vaciamiento ganglionar y bocios endotorácicos; en estos casos se habla de nervio en riesgo?

La incidencia de lesión del NLR es mayor en centros no especializados, pero generalmente se acepta una frecuencia promedio de parálisis temporal después de la tiroidectomía de menos del $10.9 \%$. Por otro lado, la tasa de parálisis definitiva en equipos experimentados y con mayor casuística es del $0.3-0.5 \%$, y hasta del $3 \%$ si hay nervio en riesgo 7 .

El objetivo de este estudio fue analizar la eficacia de la neuromonitorización en la cirugía de tiroides y comparar las tasas de lesión del NLR con y sin el uso de neuromonitorización.

\section{Método}

Se realizó un estudio observacional, descriptivo y retrospectivo, en el que se analizaron los resultados de la neuromonitorización intraoperatoria intermitente del NLR en pacientes operados por enfermedad tiroidea durante los años 2012-2018. Todos los casos fueron operados por el mismo equipo de cirujanos especializados en cirugía endocrina y en todos se realizó la misma técnica quirúrgica. En los pacientes en los que se usó neuromonitorización, la intubación orotraqueal se realizó con electrodos en la superficie de la marca Medtronic ${ }^{\oplus}$; en los casos en que no se usó, se colocó un tubo endotraqueal normal. 
Consideramos lesión definitiva cuando se observó parálisis de algunas cuerdas vocales (posición paramediana de algunas de cuerdas vocales) por laringoscopia indirecta después de 6 meses de la intervención. Todos los pacientes fueron visitados en las consultas 1 semana, 1 mes y 6 meses después de la cirugía.

\section{Técnica quirúrgica}

Los pacientes fueron colocados en posición supina e hiperextensión cervical leve. Se realizó cervicotomía anterior en corbata de Kocher, apertura de los planos y elaboración de colgajos mucocutáneos. Se abrió por la línea media sin sección de la musculatura pretiroidea. Se realizaron disección de la glándula tiroides y medialización de esta, con visualización de ambos NLR antes de la sección completa de la glándula. En los casos en que se utilizó el NIM, se verificó su funcionamiento con el equipo de neuromonitorización.

\section{Estudio estadístico}

Para el análisis estadístico utilizamos el programa SPSS versión 17. Las variables cuantitativas se describieron utilizando la media, la desviación estándar y el rango. Para las variables cualitativas se obtuvieron frecuencias absolutas, expresadas en porcentajes.

Para la comparación de la prevalencia de las lesiones se utilizó la prueba de ji al cuadrado. Para todos los análisis, el nivel de significación estadística se estableció en el $5 \%$, valor $\alpha=0.05$.

\section{Resultados}

Entre los años 2012 y 2018 fueron intervenidos 571 pacientes, todos con tiroidectomía total. De ellos, en 180 se usó el monitorización Intraoperatoria de nervio laríngeo (NIM) y en 391 no se usó. El criterio para la utilización del neuroestimulador fue, en primer lugar, disponer de él, ya que al inicio del año 2015 solo disponíamos de un número limitado, por lo que se utilizó en las tiroidectomías totales con factores de riesgo, tales como diagnóstico de cáncer de tiroides, vaciamiento ganglionar o reintervención cervical (Fig. 1).

De los 571 pacientes, el $85.3 \%$ eran mujeres. En cuanto a la edad, la media fue de 46,6 años (rango: 41.6-47.9) y la mediana fue de 47 años.

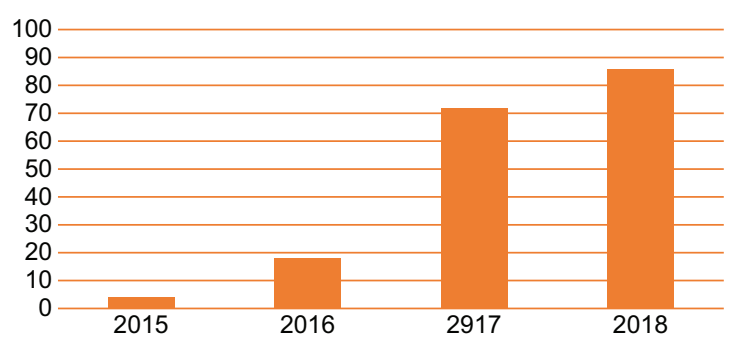

Figura 1. Evolución con el uso del neuroestimulador.

Tabla 1. Resultados de lesión del nervio laríngeo recurrente

\begin{tabular}{lccc}
\hline Parálisis & Con NIM & Sin NIM & p \\
\hline Transitoria & $8(4.4 \%)$ & $12(3 \%)$ & 0.406 \\
Definitiva & $2(1.1 \%)$ & $7(1.85 \%)$ & 0.545 \\
Total & $10(5.5 \%)$ & $19(4.85 \%)$ & \\
\hline
\end{tabular}

NIM: monitorización Intraoperatoria de nervio laríngeo.

Tabla 2. Resultados en parálisis definitivas

\begin{tabular}{lll}
\hline Parálisis definitivas & Con NIM & Sin NIM \\
\hline Nervio de alto riesgo & $2(1.1 \%)$ & \\
Nervio de bajo riesgo & $4(1.02 \%)$ & $3(0.77 \%)$ \\
\hline NIM: monitorización Intraoperatoria de nervio laríngeo.
\end{tabular}

NIM: monitorización Intraoperatoria de nervio laríngeo

Si analizamos la incidencia de parálisis transitoria en los dos grupos, 20 pacientes (3.4\%) y 9 pacientes (1.5\%) presentaron parálisis definitiva. Analizando los grupos por separado, con el uso de NIM hubo 8 (4.4\%) parálisis transitorias y $2(1.1 \%)$ definitivas, y sin el uso de NIM hubo 12 (3\%) parálisis transitorias y 7 (1.85\%) definitivas. Si comparamos ambos grupos de manera estadística, obtenemos que no hay una diferencia estadísticamente significativa en las parálisis transitorias y definitivas (Tabla 1).

Cuando analizamos las lesiones definitivas por subgrupos, vemos que las dos parálisis definitivas con el uso de NIM fueron por tiroidectomía total con vaciamiento central por carcinoma papilar (nervio de riesgo), y las lesiones definitivas sin usar NIM fueron en tres tiroidectomías totales por carcinoma papilar (nervio de riesgo), una tiroidectomía total con esternotomía para bocio endotorácico y tres tiroidectomías totales para bocio multinodular (Tabla 2). 
Los pacientes fueron revisados en consultas $1 \mathrm{se}$ mana, 1 mes y 6 meses después de la cirugía, sin que se hallaran diferencias entre el uso o no de neuroestimulación en la recuperación posterior.

\section{Discusión}

La neuromonitorización no se ha universalizado debido a su costo inicial, pero se convierte en una ayuda técnica indispensable en centros especializados en cirugía de tiroides ${ }^{7,8}$.

La neuromonitorización intraoperatoria realizada de forma intermitente permite la construcción del mapa de localización del NLR durante la ejecución de la técnica quirúrgica, de forma complementaria a la identificación anatómica obligatoria del nervio durante la disección, lo que permite trazar la ruta y el camino de sus ramos (mapeo) y proporcionar una evaluación funcional.

El desafío técnico quirúrgico se produce ante la divergencia, debido al hecho de que la conservación anatómica del nervio no es equivalente a su integridad funcional, por lo que, en la práctica clínica, la parálisis puede existir a pesar de que se aprecie que el nervio está anatómicamente intacto. Esto desaparece cuando se utiliza el NIM y se observa la contracción de las cuerdas vocales, en forma de potenciales de acción.

Podemos destacar el estudio realizado por Barczyński, et al. ${ }^{9}$, en el que analizaron una muestra de 1000 pacientes en un ensayo clínico aleatorizado (500 pacientes en cada grupo) con y sin neuromonitorización. Los autores concluyen que el número de parálisis transitorias fue menor con el uso del NIM, con una diferencia estadísticamente significativa; sin embargo, no hubo diferencias en términos de parálisis definitiva (0.8 vs. $1.2 \%$ ).

Analizaron los subgrupos de bajo riesgo entre aquellos en los que no encontraron diferencias estadísticas en ninguna de las parálisis, pero lo hicieron cuando había nervios en riesgo. Este ensayo clínico aleatorizado con una muestra bastante grande respalda nuestros resultados.

En nuestro estudio podemos concluir que la neuromonitorización es útil para reducir las lesiones del NLR, tanto parálisis transitorias como definitivas. En los nervios en riesgo, la utilidad aún es mayor.

Como ya hemos descrito, en nuestro servicio, el uso del NIM se inició en 2015 y cada año se aplicó a más pacientes, hasta que prácticamente a partir de 2017 se usa de manera sistemática en los pacientes que van a someterse a una tiroidectomía total, cualquiera que sea la enfermedad de la tiroides. Además del uso intraoperatorio del neuroestimulador, habitualmente proponemos la identificación visual del NLR, que luego verificamos con el NIM.

Hay otro estudio que podemos destacar, realizado por Stark, et al..$^{10}$, que revela que el uso de neuroestimulación no puede reducir el riesgo de lesión del NLR, y que aunque es de utilidad no reemplaza la identificación visual del nervio.

En resumen, podemos decir que hay muchos estudios al respecto de la neuroestimulación, algunos que respaldan su uso sistemático y otros que analizan su uso, pero la mayoría de los autores están de acuerdo en que es una herramienta complementaria esencial en la cirugía de tiroides.

\section{Conclusiones}

Según nuestros resultados, aunque no hay diferencias estadísticamente significativas en la disminución de las lesiones transitorias y definitivas del NLR con el uso de neuromonitorización, podemos ver que hay una tendencia significativa con respecto al uso del NIM. Consideramos que la neuromonitorización intraoperatoria es de gran ayuda para la identificación del NLR, siempre complementando la identificación visual. Atendiendo a nuestros resultados, los pacientes que pueden obtener más beneficios son aquellos que presentan nervio en riesgo. Se necesitan más estudios aleatorizados para una estandarización global.

\section{Conflicto de intereses}

Los autores declaran que no tienen conflictos de intereses.

\section{Financiamiento}

Los autores declaran que no ha habido financiación de ningún patrocinador.

\section{Responsabilidades éticas}

Protección de personas y animales. Los autores declaran que para esta investigación no se han realizado experimentos en seres humanos ni en animales.

Confidencialidad de los datos. Los autores declaran que han seguido los protocolos de su centro de trabajo sobre la publicación de datos de pacientes.

Derecho a la privacidad y consentimiento informado. Los autores declaran que en este artículo no aparecen datos de pacientes. 


\section{Bibliografía}

1. Sinagra D, Montesinos M, Tacchi V, Moreno JC, Falco JE, Mezzadri NA et al. Voice changes after thyroidectomy without recurrent laryngeal nerve injury. J Am Coll Surg. 2004;199:556-60.

2. Kern K. Medicolegal analysis of errors om diagnosis and treatment of surgical endocrine disease. Surgery. 1993:114:1167-73.

3. Angelos $P$. Recurrent laryngeal nerve monitoring: Stage of art, ethical and legal issues. Surg Clin N Am. 2009;89:1157-69.

4. Higgins T, Gupta R, Ketcham A, Sataloff R, Wadsworth JT, Sinacori JT, et al. Recurrent laryngeal nerve monitoring versus identification alone on post-thyroidectomy true vocal palsy: a meta-analysis. Laryngoscope. 2011;121:1009-17.

5. Cavicchi O, Caliceti U, Fernandez I, Macrì G, Di Lieto C, Marcantoni A, et al. The value of neuroestimulation and intraoperative nerve monitoring of inferior laryngeal nerve in thyroid surgery. Otolaryngol Head Neck Surg. 2009;140:866-70.

6. Hermann M, Hellebart C, Freissmuth M. Neuromonitoring in thyroid surgery: prospective evaluation of intraoperative electrophysiological responses for the prediction of recurrent laryngeal nerve injury. Ann Surg. 2004:240:9-17.

7. Thomusch O, Sekulla C, Machens A, Neumann H, Timmermann W, Dralle H. Validity of intraoperative neuromonitoring signals in thyroid surgery. Langenbecks Arch Surg. 2004;389:499-503.

8. Chiang F, Lee K, Chen HC, Chen HY, Lu I, Kuo W, et al. Standardization of intraoperative monitoring of recurrent laryngeal nerve in thyroid operation. World J Surg. 2010;34:223-9.

9. Barczyński M, Konturek A, Cichon S. Randomized clinical trial of visualization versus neuromonitoring of recurrent laryngeal nerves during thyroidectomy. Br J Surg. 2009;96:240-6.

10. Stark T, Rosenberg D, Dazert S, Gurr A. Value of nerve monitoringin thyroid surgery. Laryngorhinootologie. 2010;89:25-28. 